\title{
Multidisciplinary team a prerequisite in endoscopic endonasal skull base surgery
}

\author{
Jan Olofsson
}

Published online: 10 January 2010

(c) Springer-Verlag 2010

In this issue, you find the article "Endoscopic endonasal skull base surgery: past, present and future" by Castelnuovo P, Dallan I, Battaglia P and Bognami M.

This is an important and valuable article that is submitted to our journal.

Endoscopic skull base surgery is under rapid and expanding development. This article focuses on the tremendous development we have experienced with this technique during the past years. The authors stress the current anatomical knowledge in a combination with computer-assisted surgery as main reasons allowing surgery to be performed beyond the nasal cavity and sinuses themselves.

Nowadays, it is well accepted to use endoscopic routes in the management of benign diseases. However, the endoscopic techniques are still debated for nasal and paranasal malignancies. The article gives a nice review of what is going on within this field of skull base surgery.

The main chapter headings, include the background history of the techniques, inflammatory pathologies, phlogistic diseases, cerebrospinal fluid leaks, orbital and lacrimal pathway pathologies, pituitary surgery, benign neoplastic lesions, sinonasal malignancies, rhinopharyngeal and infratemporal fossa surgery, extrasellar (supra, retro) lesions and cavernous sinus surgery, surgery concerning clivus and petrous apex, surgery of the craniocervical region, as well as intracranial and skull base pathologies.

The future involves the use of robotic surgery giving a three-dimensional visualisation and two-hand surgery through small incisions and openings.

J. Olofsson ( $\square)$

Bergen, Norway

e-mail: jan.olofsson@haukeland.no
There seems to be nearly unlimited possibilities in the future using all techniques available. The authors stress the importance of multidisciplinary collaboration, especially with our neurosurgical colleagues. The authors also stress the need for careful and detailed anatomical knowledge which is true not only for endoscopic endonasal surgery, but for all operative techniques. The mentioned techniques may not only be limited to transnasal, but more routes especially transorally should be exploited.

The techniques used and the operations performed need a lot of experience. There is especially a need for establishment of centres of excellence to which we in Europe can refer our patients that are in need of this special surgery. There is also a need for these centres to take a responsibility for education of the present and for the forthcoming generations for this technically fantastically interesting field of our speciality.

The endoscopic endonasal skull base surgery has opened up a new field for ORL-HNS in collaboration especially with neurosurgeons using four-hand techniques.

From an administrative and medico-legal point of view, this type of sub-, or better super-specialisation, will in the future need some kind of accreditation.

The actual article by Paolo Castelnuovo et al. definitely gives an insight into past, present and future for endoscopic endonasal skull base surgery. The article is well worth reading. The references quoted are up-to-date and give us additional possibilities to deepen our knowledge within this progressive field of surgery. 